\title{
Deconstructing NGC 7130
}

\author{
N. A. Levenson ${ }^{1}$, K. A. Weaver ${ }^{2,3}$, T. M. Heckman ${ }^{3}, H$. Awaki $^{4}$, and Y. Terashima ${ }^{5}$
}

\begin{abstract}
Observations of the Seyfert 2 and starburst galaxy NGC 7130 with the Chandra X-ray Observatory illustrate that both of these phenomena contribute significantly to the galaxy's detectable X-ray emission. The active galactic nucleus (AGN) is strongly obscured, buried beneath column density $N_{H}>10^{24} \mathrm{~cm}^{-2}$, and it is most evident in a prominent $\mathrm{Fe} \mathrm{K} \alpha$ emission line with equivalent width greater than $1 \mathrm{keV}$. The AGN accounts for most (60\%) of the observed X-rays at energy $\mathrm{E}>2 \mathrm{keV}$, with the remainder due to spatially extended star formation. The soft X-ray emission is strong and predominantly thermal, on both small and large scales. We attribute the thermal emission to stellar processes. In total, the $\mathrm{AGN}$ is responsible for only one-third of the observed $0.5-10 \mathrm{keV}$ luminosity of $3 \times 10^{41} \mathrm{erg} \mathrm{s}^{-1}$ of this galaxy, and less than half of its bolometric luminosity. Starburst/AGN composite galaxies like NGC 7130 are truly common, and similar examples may contribute significantly to the high-energy cosmic Xray background while remaining hidden at lower energies, especially if they are distant.
\end{abstract}

Subject headings: Galaxies: individual (NGC 7130) — galaxies: Seyfert — Xrays: galaxies

\section{Introduction}

Accretion onto supermassive black holes powers active galactic nuclei (AGNs), but star formation also may be fundamentally related to the central black holes and their activity.

\footnotetext{
${ }^{1}$ Department of Physics and Astronomy, University of Kentucky, Lexington, KY 40506; levenson@pa.uky.edu

${ }^{2}$ Code 662, NASA/GSFC, Greenbelt, MD 20771

${ }^{3}$ Department of Physics and Astronomy, Bloomberg Center, Johns Hopkins University, Baltimore, MD 21218

${ }^{4}$ Department of Physics, Faculty of Science, Ehime University, Bunkyo-cho, Matsuyama, Ehime 790-8577, Japan

${ }^{5}$ Institute of Space and Astronautical Science, 3-1-1 Yoshinodai, Sagamihara, Kanagawa 229-8510, Japan
} 
For example, galaxies with AGN tend to contain a significant population of recently-formed stars, and the AGN luminosity is correlated with the stellar age (Kauffmann et al. 2003). The presence of large reservoirs of gas and the dynamical conditions for star formation simultaneously fulfill the requirements for fueling an active nucleus. The underlying connection between AGN and starbursts may be more significant than merely coincidental, being truly causal. In this interpretation, the black holes that are a consequence of massive star evolution serve as the seeds of the central supermassive black hole, with subsequent stellar outflows and supernovae promoting accretion to increase mass (e.g., Weedman 1983; Norman \& Scoville 1988).

Locally, transient bursts of star formation are correlated with AGN. Among the wellstudied Seyfert 2s, the lower-luminosity obscured variety of AGN, nearly half also contain circumnuclear starbursts (Cid Fernandes, Storchi-Bergmann, \& Schmitt 1998; González Delgado, Heckman, \& Leitherer 2001). These are the starburst/AGN composite galaxies. While either star formation or accretion may dominate the luminosity and classification of a particular galaxy, the commonplace composite galaxies demonstrate that the two processes are not exclusive. Rather, both phenomena are often energetically relevant and should be considered together in observations of real galaxies.

One complication of the known starburst/AGN composite galaxies is that they are preferentially more obscured than their counterparts that lack starbursts (Levenson, Weaver, \& Heckman 2001a; Risaliti, Maiolino, \& Salvati 1999). In the extreme case where the obscuring column density $N_{H}>1.5 \times 10^{24} \mathrm{~cm}^{-2}$, which are classified as Compton thick, the AGN X-ray emission is detected only indirectly (e.g., Krolik, Madau, \& Życki 1994; Ghisellini, Haardt, \& Matt 1994). Although the X-rays of a less-obscured AGN provide an unambiguous signature of the central engine's intrinsic power, the signal of a Compton thick AGN is diminished by orders of magnitude, and the corresponding starburst can be strong, particularly at soft X-ray energies $(E<2 \mathrm{keV})$.

In nearby galaxies, however, the confusing multiple emission sources can be disentangled. With high signal-to-noise and high spatial resolution observations, the physical conditions can be determined directly and accurately. Such detailed measurements are impossible in more distant cases or surveys. The $3^{\prime \prime}$ spectroscopic fibers of the Sloan Digital Sky Survey (York et al. 2000), for example, correspond to a physical scale larger than $1 \mathrm{kpc}$ at distances greater than $70 \mathrm{Mpc}$. Nearby case studies therefore serve as the empirical building blocks of more distant and less well-studied galaxies.

We investigate the case of NGC 7130, an example of a starburst/AGN composite galaxy. Classified on the basis of optical emission line ratios, it is a normal Seyfert 2 (Phillips, Charles, \& Baldwin 1983), while most of the ultraviolet emission is spectrally characteristic of star 
formation (Thuan 1984). The circumnuclear starburst is powerful and compact (90 pc), evident in both the vacuum ultraviolet spectrum, which shows absorption features formed in the winds and photospheres of massive stars, and in the optical spectrum, where the highorder Balmer series and He I lines are observed in absorption (González Delgado et al. 1998). Reprocessed starlight is evident in the far-infrared, where NGC 7130 has been observed with the Infrared Astronomical Satellite (IRAS) and the Infrared Space Observatory (Spinoglio, Andreani, \& Malkan 2002). NGC 7130 is also a known X-ray source (Risaliti et al. 1999), with spatial extent and thermal emission indicating that the AGN alone is not responsible for the high-energy luminosity (Levenson, Weaver, \& Heckman 2001b). We illustrate here that both the starburst and the AGN are energetically important, even in the X-ray regime.

In this work, we present new observations of NGC 7130 obtained with the Chandra X-ray Observatory (Chandra; §2). Both the starburst and AGN characteristics are evident in X-ray imaging and spectroscopy (§3). The broader spectral energy distribution of NGC 7130, particularly at far-infrared wavelengths, reveals the relative bolometric contributions of star formation and the AGN separately (§4). The example of NGC 7130 demonstrates the difficulty of diagnosing AGN properties when a starburst is present, particularly when sensitivity is limited $(\S 5)$. For $H_{0}=70 \mathrm{~km} \mathrm{~s}^{-1} \mathrm{Mpc}^{-1}$, the distance to NGC 7130 is $69 \mathrm{Mpc}$, and $1^{\prime \prime} \equiv 330 \mathrm{pc}$.

\section{Observations and Image Analysis}

The Chandra Advanced CCD Imaging Spectrometer (ACIS) observed NGC 7130 on the back-illuminated S3 detector on 2001 October 23-24. (See Weisskopf et al. 2000 for more information on Chandra.) We reprocessed all data from original Level 1 event files removing the spectral and spatial randomization that is included in standard processing with Chandra Interactive Analysis of Observations (CIAO) software. We applied the current gain corrections for the S3 CCD (from 2001 July 31), and we included only good events that do not lie on node boundaries, where discrimination of cosmic rays is difficult. We examined the lightcurves of background regions and found no significant flares, so we used all data within the standard good-time intervals, for a total exposure of $38.6 \mathrm{ks}$.

In the total Chandra bandpass $(0.3-8 \mathrm{keV})$, the emission is strongly concentrated around the nucleus, and also includes a significant extended component. Figure 1a contains the broad-band image, which has been adaptively smoothed using the CIAO task csmooth. We employed the Gaussian kernel and smoothed to a minimum significance of $2 \sigma$ and a maximum scale of 5 pixels. 
Using Chandra's simultaneous spectral and spatial resolution, we show morphological differences within the X-ray bandpass. The soft $(0.3-1 \mathrm{keV}) \mathrm{X}$-ray image (Figure $1 \mathrm{~b})$ is very similar to the total image, with a strong central concentration and significant extended emission that traces the galaxy's optical structure. Several strong individual sources outside the nucleus are also evident. In a medium X-ray band (1-2 keV), the significant emission is confined to a slightly smaller spatial scale, although several particular sources or regions of enhanced emission outside the nucleus are still detected (Figure 1c). In hard X-rays (2-8 keV), the largest-scale extended emission is absent (Figure 1d), but significant diffuse emission is present, particularly north of the nucleus. The nucleus is the strongest source in the image, and an additional source is also present. In the total and individual bands, the diffuse emission northwest of the nucleus is brighter than the extended emission toward the southeast. In the soft image, for example, the diffuse emission has a mean surface brightness of 2.2 and 1.3 counts $\operatorname{arcsec}^{-2}$ in these two areas, respectively. This may be a consequence of extinction, with the foreground inclined plane of NGC 7130 absorbing some of the X-rays.

In all three energy bands, emission is certainly extended on large $\left(>5^{\prime \prime}\right)$ scales. We compare the central source with Chandra's point-spread function (PSF) in the three separate bands to determine its extent on smaller scales. If the AGN were the sole source of nuclear emission, we would expect the emission to be unresolved, for the PSF FWHM $\approx 0$ !'8, which corresponds to $270 \mathrm{pc}$ at the distance of NGC 7130. In each of the three bands, we measure the radial profile of the observed emission and compare it with the radial profile of the PSF at the appropriate energy and detector position modeled with the CIAO task mkpsf (Figure 2). In each of the data and PSF images, we construct a radial profile by associating the counts in an individual pixel with the pixel's radial distance from the central emission peak. Thus, in each case, several data points are located within fractional pixel distances of the center, within the FWHM of the PSF. A two-dimensional Gaussian fit determines the location of the central peak, but it does not accurately characterize the shape of the PSF. The individual data points measured in NGC 7130 are plotted with small filled circles. These data combined into bins of signal-to-noise $\geq 3$ and their errors are plotted as crosses. A smooth line connects the individual points measured in the PSF images.

In the soft and medium bands, the observed radial profile is significantly broader than the PSF on scales smaller than $1^{\prime \prime}$. In the hard band, where the PSF is slightly broader, the small-scale emission appears marginally resolved, but the low signal-to-noise ratio precludes a significant measurement. We conclude that the central source is resolved at least at soft and medium energies, so these broad-band emission peaks are not due to the unresolvable AGN alone.

As we demonstrate spectroscopically, the nuclear emission is due to both the AGN and 
circumnuclear star formation, while the extended emission is due to stellar processes alone. Uniform smoothing of the data indicate the largest scale of the total X-ray emission of $38 \times$ $36^{\prime \prime}(13 \times 12 \mathrm{kpc})$. The X-rays cover nearly the full extent of the galaxy's optical emission, as the X-ray contours overlaid on the Digitized Sky Survey image illustrate (Figure 3). On smaller scales the X-ray and optical emission are correlated. Several low-level X-ray peaks located more than a kpc from the nucleus are also sites of significant star formation, evident in an optical image obtained with the Hubble Space Telescope (HST; Figure 4). NGC 7130 was observed through the F606W filter using the Wide-Field and Planetary Camera 2 for 500 s on 1994 August 23 (data set U2E60W01T). We use standard processing of the optical image and shift the X-ray contours 0 '.75 southwest to approximately align the bright nuclear emission and other sources that appear at both wavelengths. This shift is typical of the expected uncertainties in the astrometry of the two observations.

\section{Spectroscopy}

We extracted spectra of the nucleus and bright extended regions separately, and we also considered a very extended region to measure the X-ray emission of the galaxy as a whole. In all cases, we measured the local background in surrounding source-free regions and subtracted it. Over the course of the Chandra mission, the soft X-ray sensitivity has diminished, likely the result of build-up of material on the detector. We have used the "ACISABS" model of G. Chatras and K. Getman ${ }^{1}$ to create ancillary response files that account for this timevarying effect. We performed the model fitting in XSPEC (Arnaud 1996). In the best-fitting models discussed below, only data from 0.4 to $8.0 \mathrm{keV}$ are considered, and the inclusion of additional model components and their free parameters are significant at a minimum of the $95 \%$ confidence limit, based on an $F$ test. All quoted errors are $90 \%$ confidence for one parameter of interest.

In all cases, the spectral models we consider here begin with contributions typical of both AGNs and starburst galaxies. The intrinsic AGN continuum observed in Seyfert 1 galaxies has a photon index $\Gamma \approx 1.9$ (Nandra \& Pounds 1994). In Seyfert 2 galaxies, photoelectric absorption diminishes the soft X-ray emission, although the intrinsic continuum can be recovered at higher energies when the obscuration along the line of sight remains below $10^{24} \mathrm{~cm}^{-2}$. In Compton thick AGNs such as NGC 7130, however, the emergent continuum is entirely reflected and is strongly reprocessed, to $\Gamma \approx 0$ (e.g., Krolik et al. 1994) within the observed energy regime. The Fe $\mathrm{K} \alpha$ fluorescence line appears at central energy $E_{c}=6.4 \mathrm{keV}$

\footnotetext{
${ }^{1}$ http://www.astro.psu.edu/users/chartas/xcontdir/xcont.html
} 
in material that is less ionized than Fe XVII, while He-like and H-like ions produce $E_{c}=6.7$ and $6.9 \mathrm{keV}$, respectively. Risaliti et al. (1999) suggested that NGC 7130 is Compton thick based on the low ratio of $2-10 \mathrm{keV} /[\mathrm{O}$ III $] \lambda 5007$ flux and indication of the Fe line in a low signal-to-noise spectrum obtained with $A S C A$. The characteristic flat high-energy spectrum and prominent $(\mathrm{EW}>1 \mathrm{keV}) \mathrm{Fe} \mathrm{K} \alpha$ in the Chandra spectrum confirm this identification (Levenson et al. 2002).

Most of the soft X-ray emission from a starburst is genuinely diffuse thermal emission, with some portion due to individual point sources. The diffuse emission, often in an galactic-scale outflowing wind (Heckman, Armus, \& Miley 1990), is spectrally soft and typically requires multiple components at different temperatures. We model these components with the "MEKAL" equilibrium plasma model in XSPEC (Mewe, Gronenschild, \& van den Oord 1985; Mewe, Lemen, \& van den Oord 1986; Liedahl et al. 1995). The integrated contribution of the unresolved point sources produces a hard continuum that dominates the starburst's X-ray emission above $\simeq 2 \mathrm{keV}$. Surveys of starburst galaxies provide empirical support for these spectral models (Dahlem, Weaver, \& Heckman 1998; Strickland et al. 2004). Detailed studies of the nearest examples also explicitly separate the point source and diffuse contributions (Griffiths et al. 2000; Weaver et al. 2002). Theoretically-motivated models of $\mathrm{X}$-ray emission from starbursts contain these general features and further distinguish the continuum spectra of various source populations (Persic \& Rephaeli 2002), although we cannot spectrally identify all the components of the synthetic spectra here.

\subsection{The Nuclear Region}

The nuclear aperture has a radius of $1^{\prime \prime} .5$. On this physical scale (500 pc), the nuclear spectral region encompasses the compact starburst, as well as the AGN. Thus, all the physical components noted above are relevant. In order to retain significant counts at energies above the Fe line, we do not bin the data and use the C-statistic (Cash 1979). The spectrum has a total of 2189 counts, and the best-fitting model includes two thermal components, two power laws, and a Gaussian emission line. All the emission is intrinsically absorbed in excess of the Milky Way column density $\left(N_{H, M W}=1.7 \times 10^{20}\right.$; Schlegel, Finkbeiner, \& Davis 1998) along the line of sight to NGC 7130. Figure 5 shows the model fit to the data, and the model parameters are listed in Table 1, with the total flux and statistical quality on the first line. The components associated with the AGN and starburst are listed separately on subsequent lines, although all parameters were fit simultaneously in the nuclear spectrum. 


\subsection{1. $A G N$}

None of the intrinsic AGN emission emerges directly from this Compton thick source. The AGN is detected only indirectly in the flat $(\Gamma=0)$ power law and the Fe line. The power law represents the reflected continuum. The hard X-rays that do emerge are not only diminished in magnitude, but they are also strongly reprocessed. Hence, we measure a very flat spectral index of this power law component that contributes to the hard X-ray flux. The buried AGN does not produce significant soft X-ray emission, even within the nuclear aperture.

In this case, we have not applied more complete physical models, such as those of Magdziarz \& Zdziarski (1995), which explicitly account for the reprocessing of the intrinsic AGN continuum. Because all of the observable emission is reprocessed, we have no sensitivity to the underlying physical conditions that determine the emission from this model, such as intrinsic continuum slope and viewing angle. In fact, the only formal difference between the flat power law we employ and the reflection model is the Fe edge near $7 \mathrm{keV}$ of the latter, but with Chandra's diminishing sensitivity at higher energies, we cannot measure this feature in the data.

The emission line at central energy $6.4 \mathrm{keV}$ is consistent with neutral iron fluorescence. The large EW requires that the fluorescing region does not share our obscured view of the AGN continuum. More exactly, this prominent emission line absolutely rules out any model

in which the AGN continuum is viewed directly through $N_{H}<10^{24} \mathrm{~cm}^{-2}$. In addition to requiring a large column density along the line of sight, the large EW constrains the covering fraction of this material. With best-fitting $\mathrm{EW}=1.8 \mathrm{keV}$, we expect the toroidal geometry of the obscuring region to have an opening angle less than about $30^{\circ}$, or equivalently, a covering fraction greater than $75 \%$ (Krolik et al. 1994; Levenson et al. 2002). Considering the uncertainty in the EW, however, with a lower limit of $1 \mathrm{keV}$, the lower limit on the covering fraction is $35 \%$.

\subsubsection{Starburst}

The weakly-absorbed power law and the two thermal components are due to the circumnuclear starburst, which dominates the soft X-ray emission of this region. The data do not constrain the photon index of the power law well, so we adopt the fixed value $\Gamma=1.8$, reasonable for the average of the X-ray binaries that produce the non-thermal X-rays of starburst galaxies. The warmer thermal component provides most of the photons, and its temperature $(k T=0.6 \mathrm{keV})$ is similar to that measured in the inner regions of starbursts (Strickland et 
al. 2004). In NGC 7130, the very soft $(E<0.8 \mathrm{keV})$ emission is extremely strong, which we model with the lower-temperature $(k T=0.1 \mathrm{keV})$ collisional plasma. While this temperature is lower than the average observed in extended starburst winds, multi-phase models of some starburst disks, such as NGC 253, do include such a cool component (Strickland et al. 2002). In a starburst galaxy, the outflowing wind of hot gas is roughly conical, extending from the compact base of star formation out of the plane of the galaxy. Most of the flux in the nuclear aperture originates at the compact, bright base of the wind. We appear to view this outflow from an intermediate angle, given both the inclination of NGC $7130\left(29^{\circ}\right.$; Whittle 1992), and the large-scale asymmetry of the diffuse soft X-ray emission.

We emphasize that the bulk of the soft emission in the nuclear region is not directly attributable to the AGN. Photoionization models completely fail to reproduce the observed broad emission complexes of the spectrum, especially those around $1 \mathrm{keV}$, which are characteristic of collisional excitation. Existing models do not completely match the observed photoionized spectrum of NGC 1068 (Kinkhabwala et al. 2002), but the empirical spectrum of this galaxy is also inadequate as a template for the soft X-ray emission of NGC 7130. The strong thermal contribution is typical of starburst galaxies and is therefore not surprising to find within the central $500 \mathrm{pc}$ of NGC 7130, which definitely contains a powerful starburst. Although NGC 7130 also hosts an active nucleus, its accretion is not the only source of X-rays.

X-ray spectra of ordinary starburst galaxies often show non-solar abundances, especially enhanced abundances of alpha elements relative to iron (Martin, Kobulnicky, \& Heckman 2002; Strickland et al. 2004). Similar to the starburst galaxies that lack AGN, we find enhanced abundances of alpha elements relative to iron. The best-fitting model of the nuclear region includes variable $\mathrm{Fe}$ abundance in the primary (warmer) thermal component. We find $Z_{\mathrm{Fe}}=0.38(+0.1,-0.09) Z_{\odot}$, with $(\mathrm{Fe} / \mathrm{H})_{\odot}=4.68 \times 10^{-5}$ (Anders \& Grevesse 1989), fixing all other abundances at their solar values. Alternatively, fixing Fe at solar abundance and allowing the common abundance of $\mathrm{O}, \mathrm{Ne}, \mathrm{Mg}, \mathrm{Si}$, and $\mathrm{Ca}$ to vary, we find $Z_{\alpha}=2.4(+0.9,-0.6) Z_{\odot}$. In this case, the temperature is unchanged $(k T=0.6 \mathrm{keV})$, although the spectral fit is slightly worse. The strongest of these lines are oxygen, so its abundance dominates the determination of $Z_{\alpha}$, with $(\mathrm{O} / \mathrm{H})_{\odot}=8.51 \times 10^{-4}$ (Anders \& Grevesse 1989). These results indicate that Type II supernovae enrich the thermal plasma, preferentially contributing $\alpha$ elements, although the measured $\alpha$-to-iron ratio is still lower than models predict for pure supernova ejecta (see Gibson, Loewenstein, \& Mushotzky 1997, and references therein).

The small nuclear aperture isolates the AGN emission, but it excludes some of the more extended, yet strong, central emission. To better measure the total flux of the extended cen- 
tral starburst, we extracted the spectrum over a 3."5 (三1.1 kpc) radius. As expected, the only significant change is an increase in the total flux, to $1.9 \times 10^{-13}$ and $2.2 \times 10^{-13} \mathrm{erg} \mathrm{cm}^{-2} \mathrm{~s}^{-1}$ in the soft $(0.5-2 \mathrm{keV})$ and hard $(2-10 \mathrm{keV})$ bands, respectively. These values represent increases over the small nuclear region of 15 and $22 \%$ of the starburst flux in these two bands. The sense of the abundance variations in the more extended central emission is the same as in the smaller region. We find $Z_{F e}=0.52( \pm 0.1) Z_{\odot}$ for $Z_{\alpha}=Z_{\odot}$, or $Z_{\alpha}=3.2(+0.8,-0.6) Z_{\odot}$ for $Z_{F e}=Z_{\odot}$.

Alternatively, we can spatially isolate the extended emission immediately outside the nucleus in an annular aperture between 1.5 and $3 . .5$ radii. The extracted spectrum is soft, with no emission above $3 \mathrm{keV}$. The starburst model, with one thermal component $[k T=0.54$ $(+0.08,-0.1) \mathrm{keV}]$ and a power law having fixed $\Gamma=1.8$, fits well $\left(\chi^{2} / \mathrm{dof}=12 / 10\right)$. The column density is not constrained, so we fix it at the Galactic value. The observed soft and hard fluxes are 2.2 and $1.7 \times 10^{14} \mathrm{~cm}^{-2} \mathrm{~s}^{-1}$, respectively. The model underpredicts some very soft $(E<0.6 \mathrm{keV})$ emission, but an additional thermal component with $k T=0.08$ $\mathrm{keV}$ is not statistically significant. In this limited region, which includes only 285 counts, no abundance variations are significant.

\subsection{Diffuse Emission}

We isolated the diffuse emission outside the nucleus in an annulus of outer radius $15^{\prime \prime}$ and inner radius $4^{\prime \prime}$, and also excluding one bright source. This spectrum is extremely soft and requires only two of the starburst components of the nuclear model: a thermal contribution and a power law. The AGN components of the flat power law and the fluorescent line are absent. Allowing the column density to be a free parameter, we find that the obscuration would be much greater than in the nuclear region $\left[N_{H}=4.3(+0.1,-0.3) \times 10^{21}\right]$, so we fix it at the Galactic value. The spectrum and model fit are shown in Figure 6, and the bestfitting parameters of this model are listed in Table 1. The temperature here is intermediate between the two temperatures of the nuclear region. Although the spectrum is soft, the nonthermal continuum is essential and accounts for the detectable hard emission. The significant non-thermal continuum in this extra-nuclear region thus supports the interpretation of the steeper nuclear power law as a starburst component.

The surface brightness of the diffuse emission is much lower than that of the nuclear region, even considering only the starburst-associated portion. Extending over a much larger area, however, the diffuse emission contributes substantially to the total stellar luminosity. In both soft and hard bands, the luminosity of the diffuse emission is $30 \%$ of the nuclear starburst luminosity. 


\subsection{Additional Sources}

We detected several additional sources in NGC 7130. The sources are detected with non-zero spatial extent using the CIAO wavdetect algorithm. We considered total (0.3-8 $\mathrm{keV})$, low-energy $(0.3-2 \mathrm{keV})$ and high-energy $(2-8 \mathrm{keV})$ images separately, but the sources of the latter two images are a subset of sources in the total-band image. In all cases, we measure the counts in a $2^{\prime \prime}$ radius aperture after subtracting a local background. These source locations and their net counts in total, soft, and hard energy bands are listed in columns 2 through 6 , respectively, of Table 2. Most of the sources are extremely soft, with nearly all counts in the soft band and specifically below $1 \mathrm{keV}$. Two exceptions are sources 3 and 4 . Source 3 is evident in the $1-2 \mathrm{keV}$ image (Figure 1c) north of the nucleus. Source 4 is located southeast of the nucleus, most prominent in the hard X-ray image (Figure 1d), but also obvious in Figure 1c.

To estimate fluxes and luminosities, we model each spectrum as an absorbed power law with fixed $\Gamma=1.8$ and free column density in excess of the Milky Way absorption along the line of sight. While this may not be a realistic model for these sources, the procedure yields consistent results for the observed fluxes and luminosities, which we list in columns 7 and 8 of Table 2. To minimize errors due to the model-dependent estimate of fluxes and luminosities, we quote these values in the $0.3-8 \mathrm{keV}$ band and do not correct the luminosity for absorption.

Each source measurement covers a region of $650 \mathrm{pc}$ scale and could include clusters of stars; these are not necessarily individual X-ray sources. The more luminous $(L>$ $10^{39} \mathrm{erg} \mathrm{s}^{-1}$ ) sources are intriguing, however, because they exceed the Eddington luminosity of a stellar-mass black hole. If they are individual sources, they could accrete at a higher rate, emit anisotropically, or be more massive than a single neutron star. (See Miller \& Colbert 2004 and van der Marel 2004 for reviews.)

\section{Luminosity Contributions and Spectral Energy Distributions}

We list various contributions to the observed luminosity in Table 3. To calculate the luminosity of NGC 7130 as a whole, we have examined the spectrum of the $38^{\prime \prime}$ (13 kpc) emission extent. This region obviously encompasses a range of physically distinct emission sources but provides a reasonable estimate of the total flux. The AGN and starburst components of the bright central region remain strongest, with the temperature of the dominant thermal component slightly lower $(0.56 \mathrm{keV})$ than its counterpart in the nuclear spectrum. The AGN contribution listed in the table includes only the flat power law and Fe line mea- 
sured in the small nuclear aperture. The central starburst includes the thermal emission and power law of the larger (3".5) nuclear aperture. The extended starburst includes only the emission outside the nucleus yet within the central $30^{\prime \prime}$, excluding the bright source 4. The total galactic emission exceeds the sum of individual regions because this aperture covers a larger area. The gaps between the individual interior apertures serve to isolate physically-distinct regions, and individual sources are excluded from the diffuse region.

In the integrated spectrum, the soft and hard X-ray emission are comparable, yet they each have a distinct origin. Nearly all of the detected soft X-rays are due to the central starburst, with the AGN directly accounting for almost none (1\%) of the total soft emission. The AGN is responsible for most (60\%) of the detected hard X-rays, though an appreciable fraction $(40 \%)$ of the total hard flux is stellar.

The relative contributions of the AGN and star formation are also evident in spectral energy distributions (SEDs) of NGC 7130. On large scales, the observed emission of NGC 7130 is very similar to ordinary starburst galaxies at most energies. In Figure 7, a rough scaling of the median reddened starburst, which Schmitt et al. (1997) determined empirically, passes through most of measurements of NGC 7130 on scales larger than $2^{\prime \prime}$. The starburst SED is extended to the X-rays using the relationships of Ranalli, Comastri, \& Setti (2003). The published data are from Spinoglio et al. (2002), the IRAS Faint Source Catalog (Moshir et al. 1990), the 2MASS Extended Object Catalog, Storchi-Bergmann, Kinney, \& Challis (1995), and Kinney et al. (1993). The extended X-ray measurements are integrated over the full $13 \mathrm{kpc}$ galactic diameter and exclude the spectrally-identified AGN components. Even at the low resolution plotted in the SED, the strong thermal contribution at $1 \mathrm{keV}$ is obvious.

The majority of the luminosity emerges at far-infrared (FIR) wavelengths, and the spectral shape of this regime is characteristic of star-forming galaxies. While active galaxies have typical 25- to 60- $\mu$ m flux ratios $f_{25} / f_{60}>0.26$ (de Grijp, Lub, \& Miley 1987), in NGC $7130 f_{25} / f_{60}=0.13$. The FIR luminosity $L_{F I R}=4.9 \times 10^{44} \mathrm{erg} \mathrm{s}^{-1}$, and the total $8-1000 \mu \mathrm{m}$ luminosity $L_{I R}=9.0 \times 10^{44} \mathrm{erg} \mathrm{s}^{-1}$, following the prescriptions of Helou, Soifer, \& RowanRobinson (1985) and Sanders \& Mirabel (1996) to calculate these luminosities from IRAS fluxes. In total, the large-scale emission of NGC 7130 is primarily stellar.

Overall, the relationship between FIR and X-ray emission is typical of star-forming galaxies, and they yield consistent estimates of the star-formation rate (SFR). In starburst galaxies, $L_{b o l} \approx L_{I R}$, so for $S F R=L_{b o l} / 2.8 \times 10^{43}$ (Leitherer \& Heckman 1995), we find $S F R=32 M_{\odot} \mathrm{yr}^{-1}$. This total SFR is consistent with the empirical X-ray scaling of Ranalli et al. (2003), who find $S F R=L_{0.5-2} / 4.5 \times 10^{39}$ in terms of the $0.5-2 \mathrm{keV}$ luminosity. This X-ray correlation yields $S F R=34 M_{\odot} \mathrm{yr}^{-1}$ over NGC 7130 as a whole, where we exclude the AGN contribution to the soft X-ray luminosity. The spatial concentration of the starburst's 
X-ray flux indicates that most of the star formation is centrally concentrated, with $70 \%$ occurring in the central $1.1 \mathrm{kpc}$ region.

The correlation of the SFR with $2-10 \mathrm{keV}$ luminosity $\left(S F R=L_{2-10} / 5.0 \times 10^{39}\right.$; Ranalli et al. 2003) yields the lower value of $S F R=12 M_{\odot} \mathrm{yr}^{-1}$, again excluding the AGN contribution to $L_{2-10}$. As Persic et al. (2004) note, the luminosity of young populations alone should reflect the current SFR. In NGC 7130, any such "correction" to the starburst's hard X-ray luminosity would further underestimate the SFR compared with the IR-determined value. We conclude that contributions from older populations (namely low-mass X-ray binaries) to the stellar $L_{2-10}$ are minimal. Instead, current star formation, including high-mass X-ray binaries and ultra-luminous X-ray sources (Colbert et al. 2004), dominate the hard X-ray luminosity of the NGC 7130 starburst.

We identify the specific contribution of the AGN at several wavelengths. In X-rays, we isolate the AGN spectrally in the small nuclear aperture, considering only the flat power-law continuum and fluorescent line emission components. In HST images we spatially isolate the AGN. In addition to the optical data described above, NGC 7130 was observed for 1,984 s

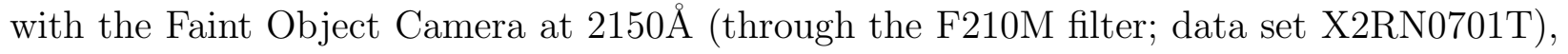
and for $320 \mathrm{~s}$ with NICMOS at $1.6 \mu \mathrm{m}$ (through the F160W filter; data set N3ZB25010). González Delgado et al. (1998) identify the nucleus with a knot of faint UV emission. This is a bright near-infrared (NIR) source, and two dust lanes converge at this location in both the optical and NIR images. For consistency, we determine the nuclear flux the same way in all three HST images. We measure the background-subtracted source flux in a small aperture to minimize contamination from nearby sources, then correct for the flux that falls outside the aperture using models of the point spread function ${ }^{2}$. In the NIR, optical, and UV bands, we measure unresolved flux densities of $8.2 \times 10^{-4}, 9.5 \times 10^{-5}$, and $4.5 \times 10^{-6} \mathrm{Jy}$, respectively.

In total, the AGN contributes very little to the observed luminosity from $1 \mathrm{~mm}$ to $10 \mathrm{keV}$. The agreement between the FIR/X-ray ratio of starburst galaxies and the observed quantities demonstrates that the AGN cannot be dominant contribution to the bolometric luminosity, either, because the reprocessed radiation would emerge in the FIR. We roughly estimate the intrinsic luminosity of the active nucleus from the X-ray observations and models. The Fe K $\alpha$ luminosity is related to the intrinsic AGN 2-10 keV luminosity, $L_{2-10, A G N}$. For the measured EW, we estimate $L_{2-10, A G N}=1 \times 10^{43} \mathrm{erg} \mathrm{s}^{-1}$ from Monte Carlo simulations of Compton thick reprocessing (Krolik et al. 1994; Levenson et al. 2002). Scaling by the empirical measurements of radio quiet quasars (Elvis et al. 1994), we find for the bolometric luminosity of the AGN below $10 \mathrm{keV} L_{b o l, A G N}=4 \times 10^{44} \mathrm{erg} \mathrm{s}^{-1}$. With the observed $L_{I R}=9.0 \times 10^{44} \mathrm{erg} \mathrm{s}^{-1}$, we

\footnotetext{
${ }^{2}$ http://www.stsci.edu/software/tinytim
} 
conclude that the AGN provides less than half the bolometric luminosity of NGC 7130.

In its total SED, NGC 7130 is similar to other galaxies that contain a Compton thick AGN along with a strong starburst. In some examples, such as Arp 299 (Della Ceca et al. 2002) and NGC 4945 (Iwasawa et al. 1993; Done, Madejski, \& Smith 1996), the AGNs are identified exclusively at X-ray energies, and the starbursts truly dominate the total emission. Observations at energies greater than $10 \mathrm{keV}$ effectively reveal these AGNs, but the bolometric luminosities of the active nuclei are only one-tenth of the totals measured in the FIR. For Arp 299, $L_{2-10, A G N}=5.6 \times 10^{42} \mathrm{erg} \mathrm{s}^{-1}$ (Della Ceca et al. 2002), or $L_{b o l, A G N}=1.7 \times$ $10^{44} \mathrm{erg} \mathrm{s}^{-1}$ (at a distance of $47.5 \mathrm{Mpc}$ for $H_{0}=70 \mathrm{~km} \mathrm{~s}^{-1} \mathrm{Mpc}^{-1}$ ). Using the integrated FIR flux densities of Soifer et al. (1989), $L_{I R}=2.9 \times 10^{45} \mathrm{erg} \mathrm{s}^{-1}$. For NGC 4945, $L_{2-10, A G N}=3 \times$ $10^{41} \mathrm{erg} \mathrm{s}^{-1}$ (Guainazzi et al. 2000), $L_{b o l, A G N}=1.1 \times 10^{43} \mathrm{erg} \mathrm{s}^{-1}$, and $L_{I R}=1.0 \times 10^{44} \mathrm{erg} \mathrm{s}^{-1}$ (Rice et al. 1988). We note that the luminosities we quote for NGC 4945 differ from those of Guainazzi et al. (2000), who claim that the AGN emission predominates. The most significant difference is that here we consistently use the $3.7 \mathrm{Mpc}$ distance to NGC 4945 (Mauersberger et al. 1996), rather than the luminosity distance of $11 \mathrm{Mpc}$ that Guainazzi et al. (2000) apply in their X-ray calculations.

Spectroscopy over large areas that is limited to $E<10 \mathrm{keV}$ does not yield accurate measurements of these AGNs in starburst-dominated galaxies. Done et al. (2003) demonstrate for NGC 4945 that most of the observed 2-10 keV flux in the roughly 1 degree $^{2}$ apertures of the Rossi X-Ray Timing Explorer instruments is not the nuclear emission that Chandra resolves. Lack of spatial discrimination likely accounts for the unusually small Fe K $\alpha \mathrm{EW}$ of Arp 299. In an XMM-Newton aperture of $8.6 \mathrm{kpc}$ diameter, Ballo et al. (2004) find EW $=422 \mathrm{eV}$ in NGC 3690, the host of the the heavily absorbed nucleus of the Arp 299 merger. High spatial resolution data from Chandra show that only $15 \%$ of the observed $2-10 \mathrm{keV}$ flux is due to this nucleus (Zezas, Ward, \& Murray 2003), but these data are not sensitive enough to determine the line EW against the nuclear continuum alone. Instead, measured against the combined continuum of an ensemble of sources, the line appears to be very weak compared with most Compton thick AGNs, where $\mathrm{EW} \geq 1 \mathrm{keV}$ is typical.

\section{Implications for Surveys}

NGC 7130 is an instructive example of the consequences of star formation and Compton thick obscuration for X-ray surveys to identify AGN. When the active nucleus is the only source of X-ray emission, the simple measure of a hardness ratio of just two energy bands characterizes it well. Especially when the obscuration is Compton thin and some of its intrinsic power is directly detected, a hardness ratio provides a good estimate of both the 
intrinsic AGN luminosity and the obscuring column density. Compton thick obscuration and coincident starbursts both complicate this simple scenario, however, even in nearby examples. Because of the stellar component, the total soft X-ray emission of NGC 7130 is very strong. If the hardness ratio is calibrated against an absorbed power law to model the X-ray emission of an AGN alone, NGC 7130 would mistakenly appear to be completely unobscured, and the intrinsic luminosity of the buried central engine would be severely underestimated.

We can use NGC 7130 to extend our analysis to larger surveys. We develop a general diagnostic using X-ray colors to distinguish between the starburst contribution and obscuration. We define soft $(0.3-2 \mathrm{keV})$, medium $(2-5 \mathrm{keV})$, and hard $(5-8 \mathrm{keV})$ energy ranges. To identify the discriminating characteristics, we consider variations of AGN obscuration and starburst strength in a composite system. In this case, the AGN is modeled as a single power law, with $\Gamma=1.9$, and column density ranging from $N_{H}=10^{22}$ to $N_{H}=10^{24} \mathrm{~cm}^{-2}$. The starburst is modeled after the central region of NGC 7130, with a strong 0.6-keV thermal plasma, a fainter soft component, and a $\Gamma=1.8$ power-law continuum. We combine each AGN with a starburst whose strength varies from 0 to $100 \%$ of the total counts. We plot the results (Figure 8) in terms of the percentage of hard and soft counts Chandra would detect, so these spectral ratios can be utilized without detailed modeling.

In general, the three broad X-ray bandpasses distinguish among a range of starburst and AGN properties. Some confusion remains in the combined cases of high obscuration and large starburst fraction, where the hard count rate is low. No discrimination is possible when the fraction of hard counts is less than about $2 \%$, which occurs for a starburst fraction in excess of about $90 \%$. With some significant hard X-ray emission, the AGN of NGC 7130 would at least be characterized as strongly obscured, although the X-ray "colors" alone do not identify it certainly as a Compton thick case.

At high redshift, however, low flux prevents even X-ray identification of an active nucleus similar to that of NGC 7130. If NGC 7130 were located at $z=1$, the $0.5-2$ and $2-10 \mathrm{keV}$ observed fluxes would be $3 \times 10^{-18}$ and $1 \times 10^{-17} \mathrm{erg} \mathrm{cm}^{-2} \mathrm{~s}^{-1}$, respectively. At $z=2$, the observed fluxes would be an order of magnitude lower. Despite the benefit of the high-energy emission that is redshifted into the observable bandpass, such Compton thick Seyferts would be undetectable, even in the deepest current surveys. While these surveys successfully resolve most of the X-ray background below $10 \mathrm{keV}$ (e.g., Mushotzky et al. 2000; Brandt et al. 2001; Giacconi et al. 2001; Hasinger et al. 2001), their sources do not successfully reproduce the spectrum at higher energies. A population of Compton thick AGN is essential (e.g., Setti \& Woltjer 1989; Comastri et al. 1995), in addition to the X-ray sources that are observed directly. The example of NGC 7130 demonstrates why this obscured class is not evident from X-ray measurements below $10 \mathrm{keV}$ alone. 
Although Compton thick AGN are missing from current X-ray surveys, the existence of this population does not conflict with current results on black hole demographics or multiwavelength background measurements. The host galaxies are observed and included in direct estimates of black hole density. These sources may be evident in the similar luminosity functions of low-luminosity AGN and star-forming galaxies. The evolution of AGNs is a function of luminosity, with the density of lower-luminosity AGNs peaking at lower redshift $(z<1$; Cowie et al. 2003; Ueda et al. 2003), similar to IR-bright starburst galaxies (Chary \& Elbaz 2001). The starburst-dominated examples, including Arp 299 and NGC 4945, explicitly demonstrate that these two classes have some members in common. Future, more sensitive missions, such as the X-ray Evolving Universe Spectrometer, with planned flux limits of $10^{-17} \mathrm{erg} \mathrm{cm}^{-2} \mathrm{~s}^{-1}$, will be able to measure these obscured AGNs at their dominant epoch.

\section{Conclusions}

Nearby Seyfert/starburst composite galaxies allow detailed examination of high signalto-noise observations. Locally, the composite galaxies are common rather than exceptional, and they serve as examples of the complications that cannot feasibly be disentangled in more distant sources. In this case study of NGC 7130, we discern the multiple physical processes that produce its intrinsic luminosity. Overall, star formation dominates the 0.3-8 keV luminosity, with both a powerful concentrated circumnuclear starburst and an extended diffuse disk component together accounting for $70 \%$ of the detectable X-rays. The AGN produces the majority of the observed emission only at higher energies, above $3 \mathrm{keV}$. The effects of obscuration and reprocessing vary with observed wavelengths and determine the emergent, observable emission. The obscuration of the nucleus of NGC 7130 is Compton thick, preventing direct detection of the intrinsic emission in the Chandra bandpass, below $8 \mathrm{keV}$. The stellar light is efficiently reprocessed to FIR wavelengths, where it provides the bulk of the bolometric luminosity.

The example of NGC 7130 empirically demonstrates that an optically-evident AGN can be hidden or confused, even at X-ray energies. Integrating over large spatial scales, the properties of the AGN would be misidentified, although broad-band flux measurements can be used to characterize the nucleus roughly. If its host galaxy were more distant, the active nucleus would not be detected at all. These buried AGNs are directly relevant to the study of the cosmic X-ray background, and NGC 7130 illustrates the difficulty of finding them.

NGC 7130 and similar galaxies are also relevant to the study of ultraluminous infrared galaxies. At issue in this class is the underlying energy source, which may be an active 
nucleus or star formation. While the local composite galaxies are not luminous enough to qualify as direct analogs of the more distant and powerful ultraluminous galaxies, they illustrate the component building blocks that may be present in the latter. The investigation of NGC 7130 also suggests methods to discriminate star formation from accretion activity, with the conclusion that over large physical scales, star formation can dominate even when a normal active nucleus is present.

We thank the referee, R. Della Ceca, for useful suggestions. This research has made use of the NASA/IPAC Extragalactic Database (NED) which is operated by the Jet Propulsion Laboratory, California Institute of Technology, under contract with the National Aeronautics and Space Administration. The Digitized Sky Survey was produced at the Space Telescope Science Institute under U.S. Government grant NAG W-2166. The image presented here was made by the Royal Observatory Edinburgh with the UK Schmidt Telescope. Part of this work was based on observations made with the NASA/ESA Hubble Space Telescope, obtained from the data archive at the Space Telescope Science Institute. STScI is operated by the Association of Universities for Research in Astronomy, Inc. under NASA contract NAS 5-26555. This publication makes use of data products from the Two Micron All Sky Survey, which is a joint project of the University of Massachusetts and the Infrared Processing and

Analysis Center/California Institute of Technology, funded by the National Aeronautics and Space Administration and the National Science Foundation. This research was supported by NASA through grant GO1-2119 and NSF CAREER award AST-0237291.

\section{REFERENCES}

Anders, E., \& Grevesse, N. 1989, Geochim. Cosmochim. Acta, 53, 197

Arnaud, K. A. 1996, in ASP Conf. Ser. 101, Astronomical Data Analysis Software and Systems V, ed. G. Jacoby \& J. Barnes (San Francisco: ASP), 17

Ballo, L., Braito, V., Della Ceca, R., Maraschi, L., Tavecchio, F., \& Dadina, M. 2004, ApJ, 600,634

Brandt, W. N., et al. 2001, AJ, 122, 1

Cash, W. 1979, ApJ, 228, 939

Chary, R. \& Elbaz, D. 2001, ApJ, 556, 562

Cid Fernandes, R. J., Storchi-Bergmann, T., \& Schmitt, H. 1998, MNRAS, 297, 579 
Colbert, E. J. M., Heckman, T. M., Ptak, A. F., Strickland, D. K., \& Weaver, K. A. 2004, ApJ, 602, 231

Comastri, A., Setti, G., Zamorani, G., \& Hasinger, G. 1995, A\&A, 296, 1

Cowie, L. L., Barger, A. J., Bautz, M. W., Brandt, W. N., \& Garmire, G. P. 2003, ApJ, 584, L57

de Grijp, M. H. K., Lub, J., \& Miley, G. K. 1987, A\&AS, 70, 95

Dahlem, M., Weaver, K. A., \& Heckman, T. M. 1998, ApJS, 118, 401

Della Ceca, R., et al. 2002, ApJ, 581, L9

Done, C., Madejski, G. M., \& Smith, D. A. 1996, ApJ, 463, L63

Done, C., Madejski, G. M., Życki, P. T., \& Greenhill, L. J. 2003, ApJ, 588, 763

Elvis, M., et al. 1994, ApJS, 95, 1

Ghisellini, G., Haardt, F., \& Matt, G. 1994, MNRAS, 267, 743

Giacconi, R., et al. 2001, ApJ, 551, 624

Gibson, B. K., Loewenstein, M., \& Mushotzky, R. F. 1997, MNRAS, 290, 623

González Delgado, R. M., Heckman, T., \& Leitherer, C. 2001, ApJ, 546, 845

González Delgado, R. M., Heckman, T., Leitherer, C., Meurer, G., Krolik, J., Wilson, A. S., Kinney, A., \& Koratkar, A. 1998, ApJ, 505, 174

Griffiths, R. E., Ptak, A., Feigelson, E. D., Garmire, G., Townsley, L., Brandt, W. N., Sambruna, R., \& Bregman, J. N. 2000, Science, 290, 1325

Guainazzi, M., Matt, G., Brandt, W. N., Antonelli, L. A., Barr, P., \& Bassani, L. 2000, A\&A, 356, 463

Hasinger, G., et al. 2001, A\&A, 365, L45

Heckman, T. M., Armus, L., \& Miley, G. K. 1990, ApJS, 74, 833

Helou, G., Soifer, B. T., \& Rowan-Robinson, M. 1985, ApJ, 298, L7

Iwasawa, K., Koyama, K., Awaki, H., Kunieda, H., Makishima, K., Tsuru, T., Ohashi, T., \& Nakai, N. 1993, ApJ, 409, 155 
Kauffman, G., et al. 2003, MNRAS, 346, 1055

Kinkhabwala, A. et al. 2002, ApJ, 575, 732

Kinney, A. L., Bohlin, R. C., Calzetti, D., Panagia, N., \& Wyse, R. F. G. 1993, ApJS, 86, 5

Krolik, J. H., Madau, P., \& Życki, P. T. 1994, ApJ, 420, L57

Leitherer, C., \& Heckman, T. M. 1995, ApJS, 96, 9

Levenson, N. A., Krolik, J. H., Życki, P. T., Heckman, T. M., Weaver, K. A., Awaki, H., \& Terashima, Y. 2002, ApJ, 573, L81

Levenson, N. A., Weaver, K. A., \& Heckman, T. M. 2001a, ApJ, 550, 230

Levenson, N. A., Weaver, K. A., \& Heckman, T. M. 2001b, ApJS, 133, 269

Liedahl, D. A., Osterheld, A. L., \& Goldstein, W. H. 1995, ApJ, 438, L115

Magdziarz, P. \& Zdziarski, A. A. 1995, MNRAS, 273, 837

Martin, C. L., Kobulnicky, H. A., \& Heckman, T. M. 2002, ApJ, 574, 663

Mauersberger, R., Henkel, C., Whiteoak, J. B., Chin, Y.-N., \& Tieftrunk, A. R. 1996, A\&A, 309,705

Mewe, R., Gronenschild, E. H. B. M., \& van den Oord, G. H. J. 1985, A\&AS, 62, 197

Mewe, R., Lemen, J. R., \& van den Oord, G. H. J. 1986, A\&AS, 65, 511

Miller, M. C., \& Colbert, E. J. M. 2004, Int. J. Mod. Phys. D, 13, 1

Moshir, M. et al. 1990, IRAS Faint Source Catalog (ver 2.0; Greenbelt:NASA/GSFC)

Mushotzky, R. F., Cowie, L. L., Barger, A. J., \& Arnaud, K. A. 2000, Nature, 404, 459

Nandra, K., \& Pounds, K. A. 1994, MNRAS, 268, 405

Norman, C. \& Scoville, N. 1988, ApJ, 332, 124

Persic, M. \& Rephaeli, Y. 2002, A\&A, 382, 843

Persic, M., Rephaeli, Y., Braito, V., Cappi, M., Della Ceca, R., Franceschini, A., \& Gruber, D. E. 2004, A\&A, 419, 849

Phillips, M. M., Charles, P. A., \& Bladwin, J. A. 1983, ApJ, 266, 485 
Ranalli, P., Comastri, A., \& Setti, G. 2003, A\&A, 399, 39

Rice, W., Lonsdale, C. J., Soifer, B. T., Neugebauer, G., Koplan, E. L., Lloyd, L. A., de Jong, T., \& Habing, H. J. 1988, ApJS, 68, 91

Risaliti, G., Maiolino, R., \& Salvati, M. 1999, ApJ, 522, 157

Sanders, D. B., \& Mirabel, I. F. 1996, ARA\&A, 34, 749

Schlegel, D. J., Finkbeiner, D. P., \& Davis, M. 1998, ApJ, 500, 525

Schmitt, H. R., Kinney, A. L., Calzetti, D., \& Storchi Bergmann, T. 1997, AJ, 114, 592

Setti, G., \& Woltjer, L. 1989, A\&A, 224, L21

Soifer, B. T., Boehmer, L., Neugebauer, G., \& Sanders, D. B. 1989, AJ, 98, 766

Spinoglio, L., Andreani, P., \& Malkan, M. A. 2002, ApJ, 572, 105

Storchi-Bergmann, T., Kinney, A. L., \& Challis, P. 1995, ApJS, 98, 103

Strickland, D. K., Heckman, T. M., Colbert, E. J. M., Hoopes, C. G., \& Weaver, K. A. 2004, ApJS, 151, 193

Strickland, D. K., Heckman, T. M., Weaver, K. A., Hoopes, C. G., \& Dahlem, M. 2002, ApJ, 568, 689

Thuan, T. X. 1984, ApJ, 218, 126

Ueda, Y., Akiyama, M., Ohta, K., \& Miyaji, T. 2003, ApJ, 598, 886

van der Marel, R. P. 2004, in Carnegie Observatories Astrophysics Series, Vol. 1: Coevolution of Black Holes and Galaxies, ed. L. C. Ho (Cambridge:Cambridge Univ. Press), 37

Weaver, K. A., Heckman, T. M., Strickland, D. K., \& Dahlem, M. 2002, ApJ, 576, L19

Weedman, D. W. 1983, ApJ, 266, 479

Weisskopf, M. C., Tananbaum, H. D., Van Speybroeck, L. P., \& O’Dell, S. L. 2000, Proc. SPIE, 4012, 2

Whittle, M. 1992, ApJS, 79, 49

York, D. G., et al. 2000, AJ, 120, 1579

Zezas, A., Ward, M. J., \& Murray, S. S. 2003, ApJ, 594, L31 
This preprint was prepared with the AAS LATEX macros v5.2. 
Table 1. Spectral Model Parameters

\begin{tabular}{|c|c|c|c|c|c|c|c|c|c|c|}
\hline Region & $N^{\mathrm{a}}{ }_{H}$ & $k T^{\mathrm{b}}$ & $A_{t h}^{\mathrm{c}}$ & $\Gamma$ & $A^{\mathrm{d}}{ }_{\text {pow }}$ & $E_{\text {line }}^{\mathrm{e}}$ & $E W^{\mathrm{f}}$ line & $f^{\mathrm{g}} 0.5-2$ & $f^{\mathrm{h}}{ }_{2-10}$ & $\chi^{2} / \operatorname{dof}$ \\
\hline \multirow{4}{*}{$\begin{array}{r}\text { AGN } \\
\text { Starburst }\end{array}$} & $\ldots$ & $\ldots$ & $\ldots$ & $\ldots$ & $\ldots$ & $\ldots$ & $\ldots$ & $1.7 \pm 0.3$ & $2.1_{-0.4}^{+0.5}$ & $475 / 509^{\mathrm{i}}$ \\
\hline & $1.6 \pm 0.8$ & $\ldots$ & $\ldots$ & $0.0 \mathrm{f}$ & $0.16_{-0.06}^{+0.07}$ & $6.40 \pm 0.05$ & $1.8_{-0.8}^{+0.7}$ & $0.04 \pm 0.02$ & $1.6_{-0.4}^{+0.4}$ & .. \\
\hline & $1.6 \pm 0.8$ & $0.08_{-0:}^{+0.01}$ & $17_{-10}^{+25}$ & $1.8 \mathrm{f}$ & $1.4 \pm 0.7$ & $\ldots$ & $\ldots$ & $1.6_{-0.2}^{+0.3}$ & $0.6 \pm 0.3$ & $\cdots$ \\
\hline & $\ldots$ & $0.61_{-0.05}^{+0.04}$ & $1.4 \pm 0.4$ & $\ldots$ & $\ldots$ & $\cdots$ & $\cdots$ & $\ldots$ & $\ldots$ & $\cdots$ \\
\hline Diffuse & $0.2 \mathrm{f}$ & $0.40 \pm 0.05$ & $0.24_{-0.05}^{+0.02}$ & $1.8 \mathrm{f}$ & $0.61_{-0.1}^{+0.3}$ & $\ldots$ & $\ldots$ & $0.62 \pm 0.06$ & $0.21 \pm 0.07$ & $40 / 34$ \\
\hline
\end{tabular}

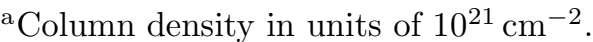

${ }^{\mathrm{b}}$ Temperature of thermal plasma in $\mathrm{keV}$.

${ }^{\mathrm{c}}$ Normalization of thermal component in units of $10^{-4} K$, where $K=\left(10^{-14} /\left(4 \pi D^{2}\right)\right) \int n_{e} n_{H} d V, D$ is the distance to the source (cm), $n_{e}$ is the electron density $\left(\mathrm{cm}^{-3}\right)$, and $n_{H}$ is the hydrogen density $\left(\mathrm{cm}^{-3}\right)$.

${ }^{\mathrm{d}}$ Normalization of power law in units of $10^{-5}$ photons $\mathrm{keV}^{-1} \mathrm{~cm}^{-2} \mathrm{~s}^{-1}$ at $1 \mathrm{keV}$.

${ }^{\mathrm{e}}$ Energy of line center in $\mathrm{keV}$.

${ }^{\mathrm{f}}$ Equivalent width of line in $\mathrm{keV}$.

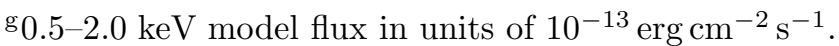

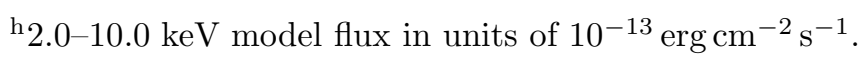

${ }^{\mathrm{i}} \mathrm{C}$-statistic.

Note. - Errors are $90 \%$ confidence limits for one interesting parameter. Parameters that are constrained by hard limits are marked with a colon. Fixed parameters are marked with $\mathrm{f}$. 
Table 2. Additional Sources

\begin{tabular}{lccrrrrr}
\hline \hline Source & $\begin{array}{c}\text { R.A. } \\
(\mathrm{J} 2000)\end{array}$ & $\begin{array}{c}\text { Decl. } \\
(\mathrm{J} 2000)\end{array}$ & $\begin{array}{c}\text { Counts } \\
(0.3-8 \mathrm{keV})\end{array}$ & $\begin{array}{c}\text { Counts } \\
(0.3-2 \mathrm{keV})\end{array}$ & $\begin{array}{c}\text { Counts } \\
(2-8 \mathrm{keV})\end{array}$ & $\begin{array}{c}f_{0.3-8} \\
\left(10^{-15} \mathrm{erg} \mathrm{cm}^{-2} \mathrm{~s}^{-1}\right)\end{array}$ & $\begin{array}{c}L_{0.3-8} \\
\left(10^{38} \mathrm{erg} \mathrm{s}^{-1}\right)\end{array}$ \\
\hline 1 & 214818.8 & -345657 & 12 & 12 & 0 & 2.7 & 16 \\
2 & 214819.4 & -345656 & 30 & 30 & 0 & 3.2 & 18 \\
3 & 214819.6 & -345658 & 15 & 13 & 2 & 6.0 & 17 \\
4 & 214819.7 & -345707 & 42 & 31 & 11 & 1.4 & 8.1 \\
5 & 214819.8 & -345717 & 12 & 10 & 2 & 1.5 & 6.5 \\
6 & 214820.6 & -345700 & 8 & 8 & 0 & 0.7 \\
7 & 214821.0 & -345711 & 8 & 8 & 0 & & 8.7 \\
\hline
\end{tabular}

Note. - Units of right ascension are hours, minutes, and seconds, and units of declination are degrees, arcminutes, and arcseconds.

Table 3. Observed Luminosities

\begin{tabular}{lcc}
\hline \hline \multicolumn{1}{c}{ Region } & $\begin{array}{c}L_{0.5-2} \\
\left(10^{40} \mathrm{erg} \mathrm{s}^{-1}\right)\end{array}$ & $\begin{array}{c}L_{2-10} \\
\left(10^{40} \mathrm{erg} \mathrm{s}^{-1}\right)\end{array}$ \\
\hline NGC 7130 (total) & 15 & 15 \\
AGN & 0.2 & 8.9 \\
Central starburst & 11 & 4.0 \\
Extended starburst & 3.5 & 1.2 \\
\hline
\end{tabular}



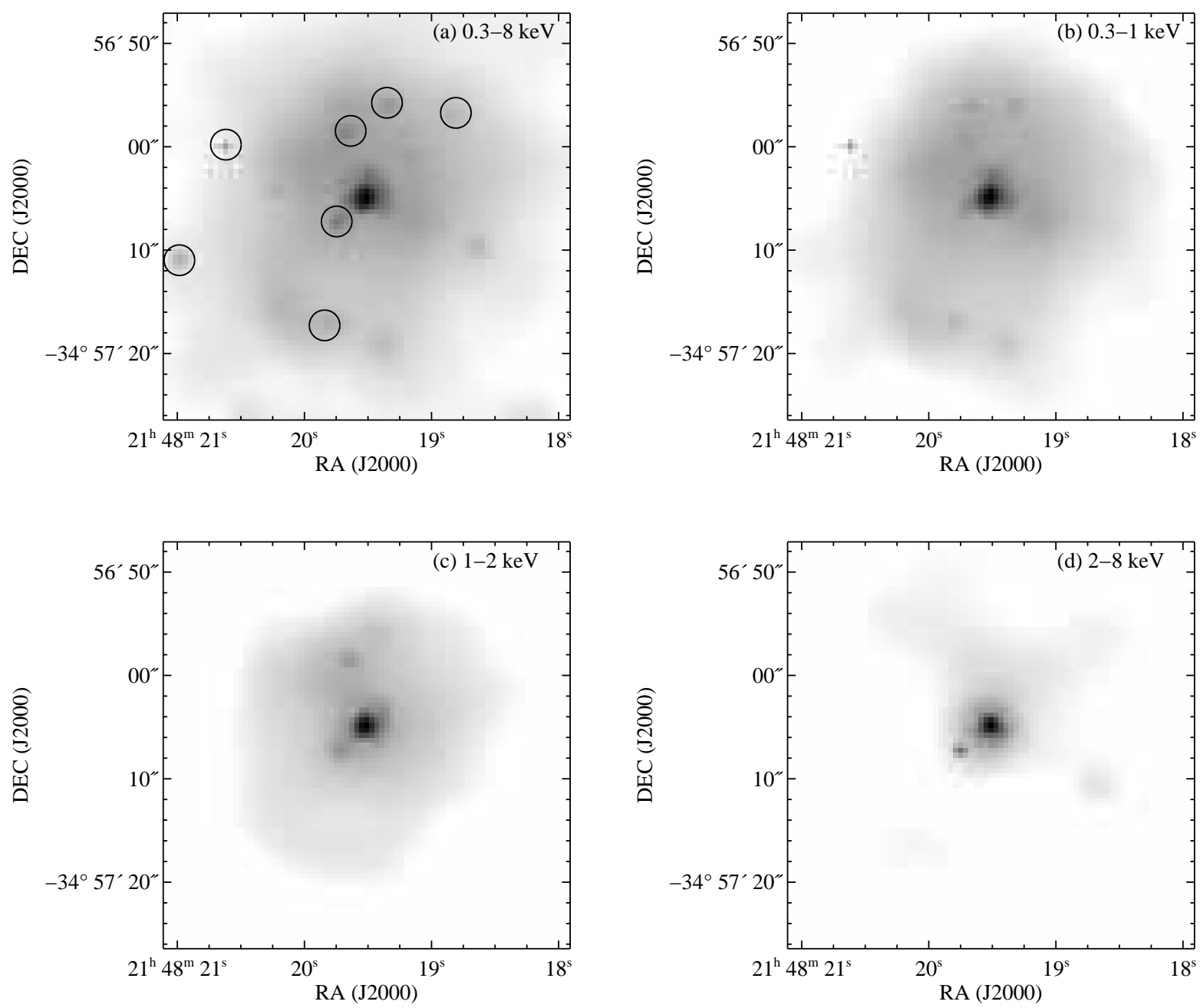

Fig. 1.- (a) Broad-band (0.3-8 keV) X-ray image of NGC 7130. The strong central concentration is due to both the AGN and the circumnuclear starburst, while the very extended emission is due to stellar processes alone. The additional extranuclear sources are marked. This image has been adaptively smoothed and is scaled logarithmically. (b) Chandra soft X-ray $(0.3-1 \mathrm{keV})$ image of NGC 7130, adaptively smoothed and scaled logarithmically. (c) Chandra medium X-ray (1-2 keV) image of NGC 7130, adaptively smoothed and scaled logarithmically. (d) Chandra hard X-ray (2-8 keV) image of NGC 7130, adaptively smoothed and scaled logarithmically. 

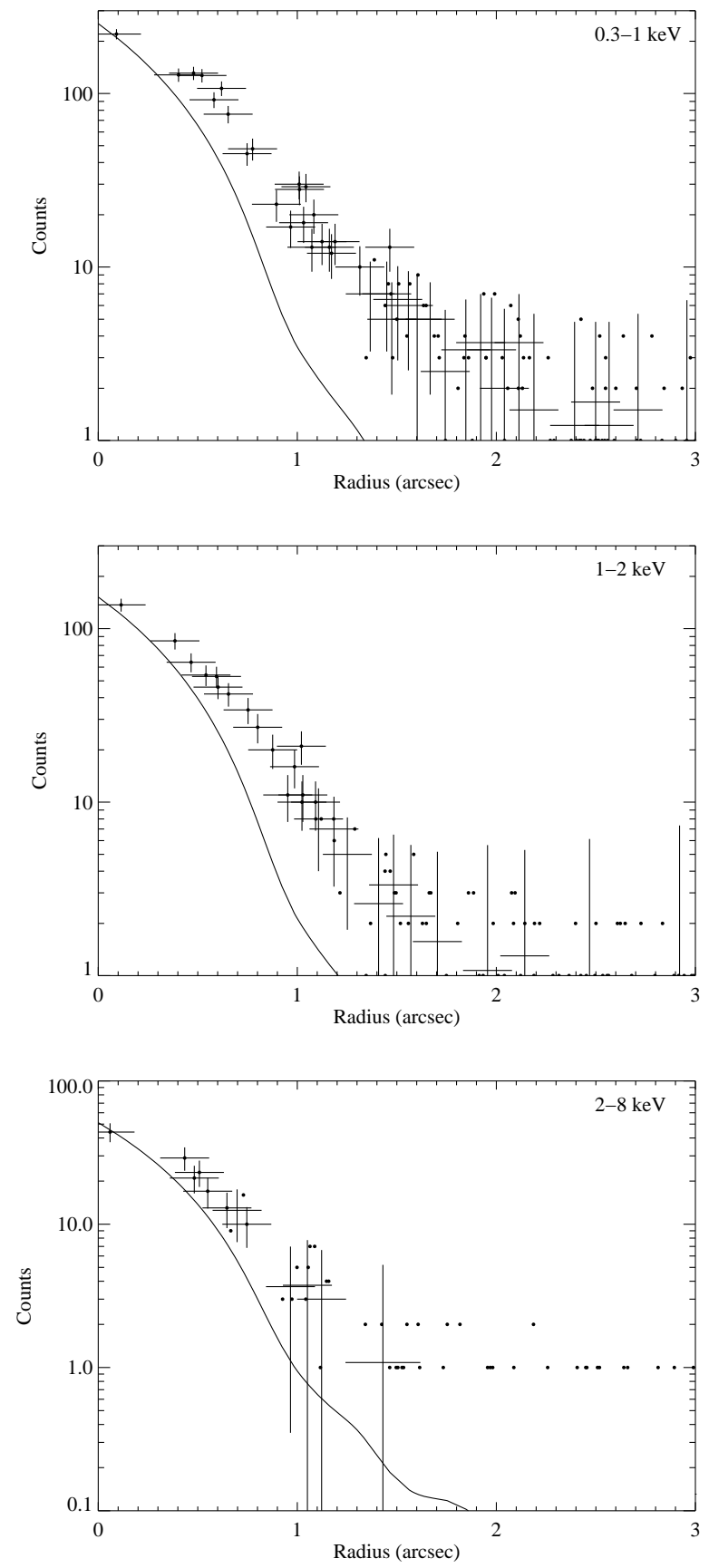

Fig. 2.- Radial profiles of the central emission at soft, medium, and hard X-ray energies. Individual data points are marked with small filled circles. Combined into bins that have a minimum signal-to-noise of 3, these data and their errors are plotted as crosses. In each case, the radial profile of the energy-appropriate PSF scaled to the central peak is plotted as a solid line. The small-scale emission is significantly resolved in the soft and medium bands. 


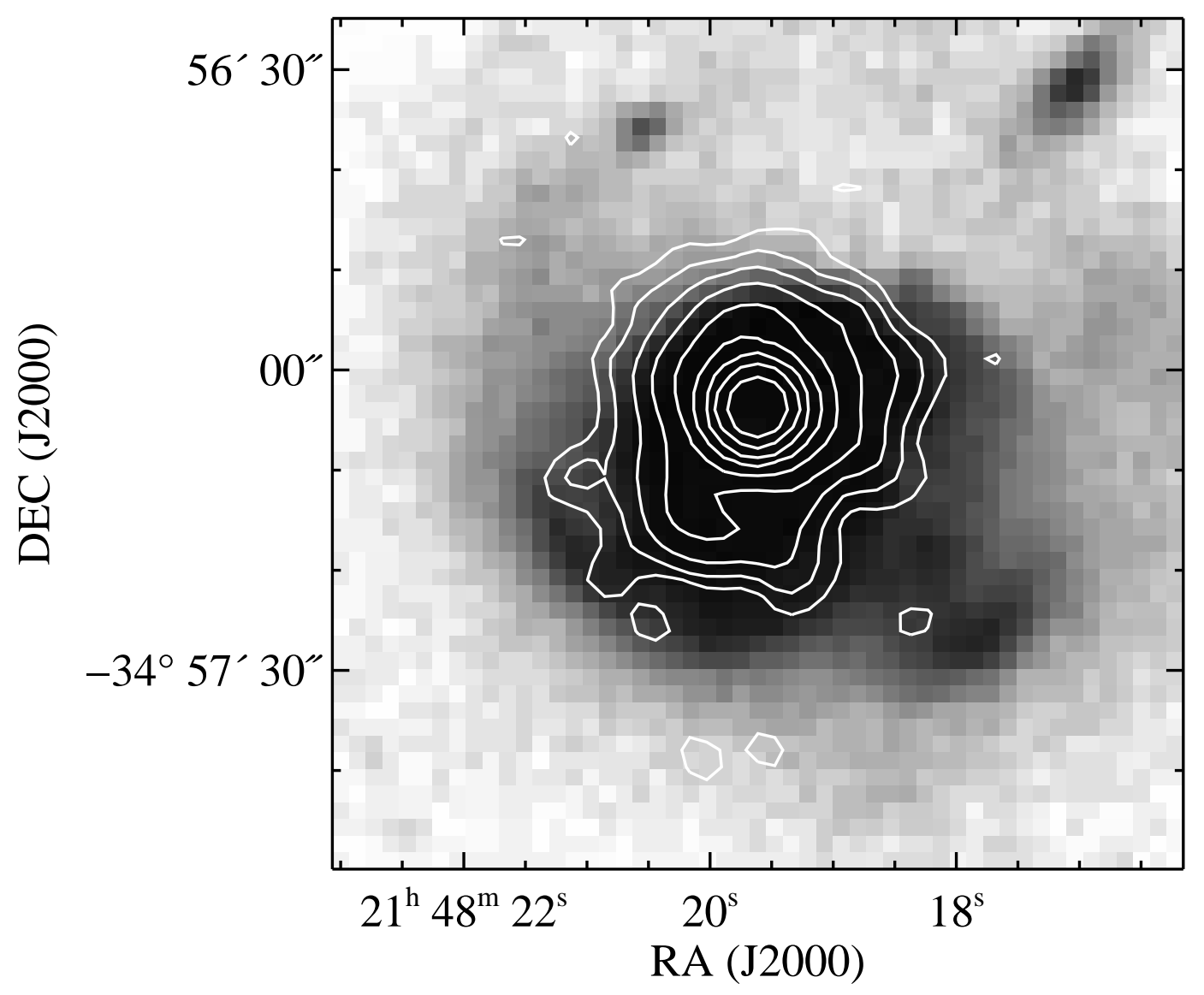

Fig. 3.- Digitized Sky Survey image with total Chandra contours overlaid. The X-ray data have been smoothed by a Gaussian of FWHM $=5^{\prime \prime}$ to illustrate the full extent of the high-energy emission, which covers most of the optically-bright galaxy. 


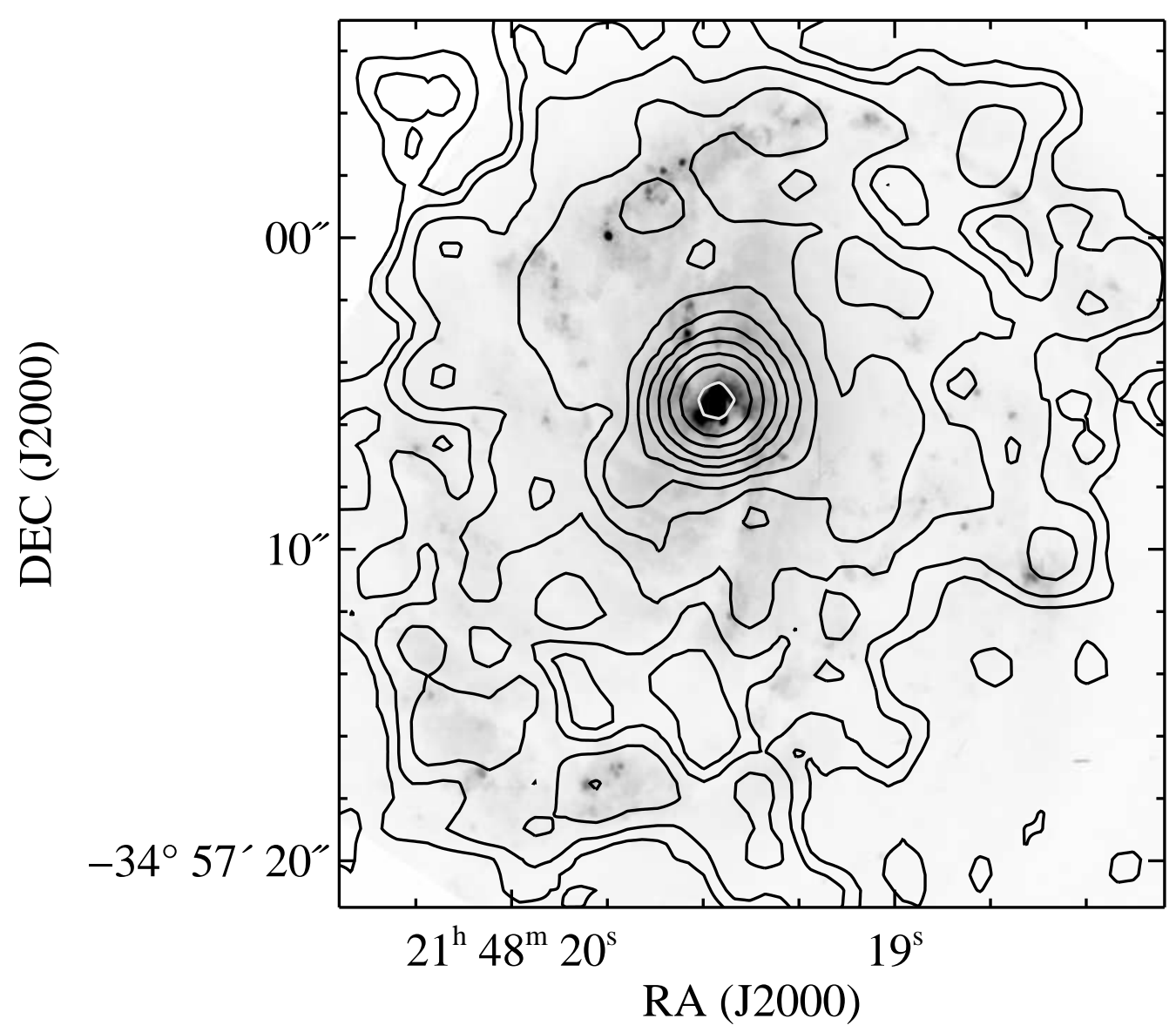

Fig. 4.- HST optical $(6030 \AA)$ image with Chandra total-band contours overlaid. The highresolution optical image shows extended stellar emission correlated with X-ray emission. Well outside the nuclear region, on kpc scales, particular sites of intense star formation are often also local X-ray enhancements. The optical image is scaled linearly, and the X-ray contours are scaled logarithmically by factors of 2 from $3 \sigma$ above the background. 

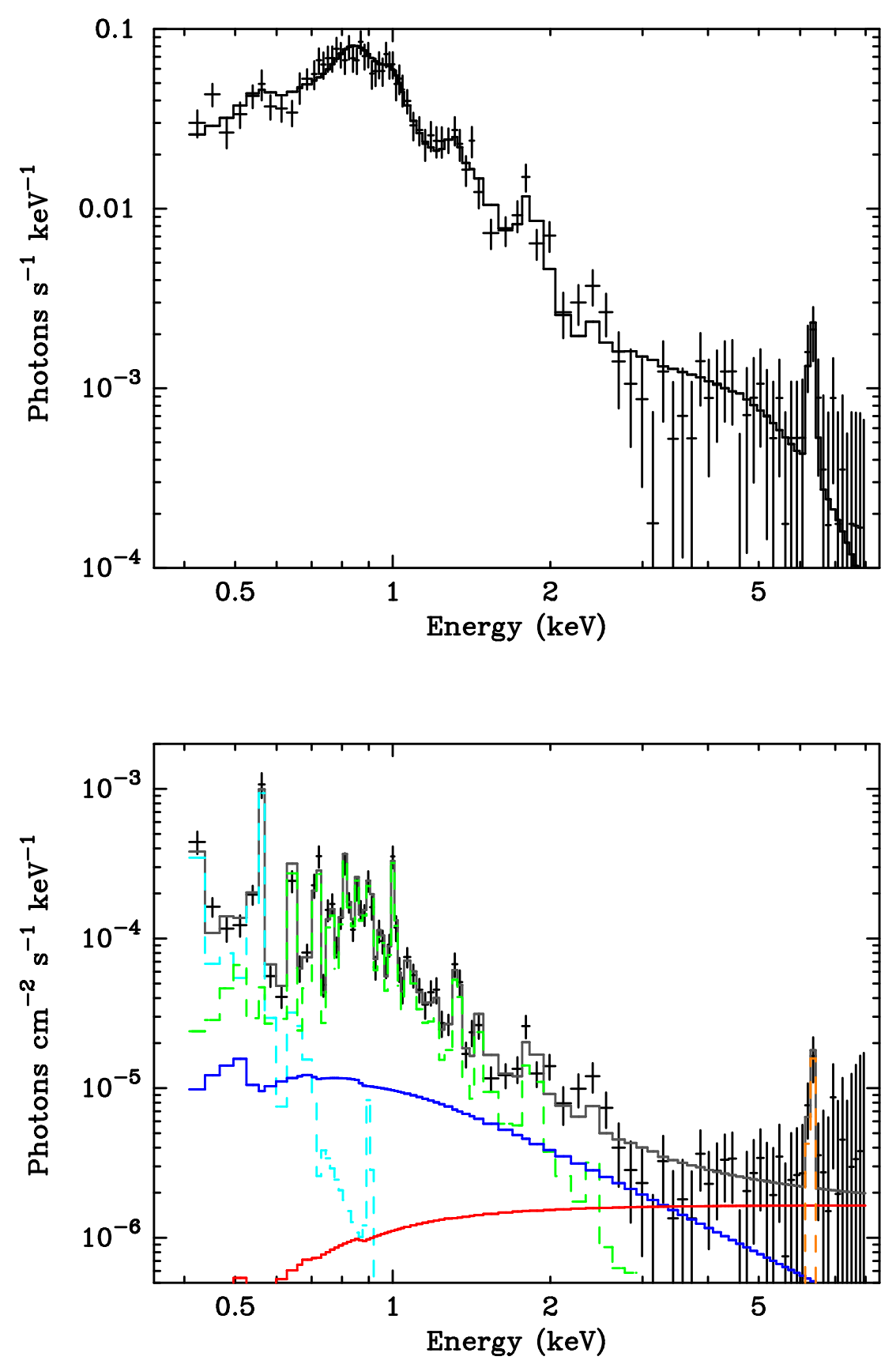

Fig. 5.- Chandra spectra of the nucleus of NGC 7130, encompassing a region of radius $1^{\prime \prime} .5 \equiv 500$ pc. The data have been rebinned so each point represents a detection that is significant at the $5 \sigma$ level, although the original unbinned data are used in the model fitting. (upper panel) Observed data (crosses) and total model convolved with detector response (histogram). (lower panel) Crosses show the intrinsic spectrum for the given model. The total model (gray histogram) comprises two thermal components (green and cyan) and a power law (blue) of the starburst, as well as the reprocessed flat power law (red) and Fe K $\alpha$ line (orange) of the AGN. 

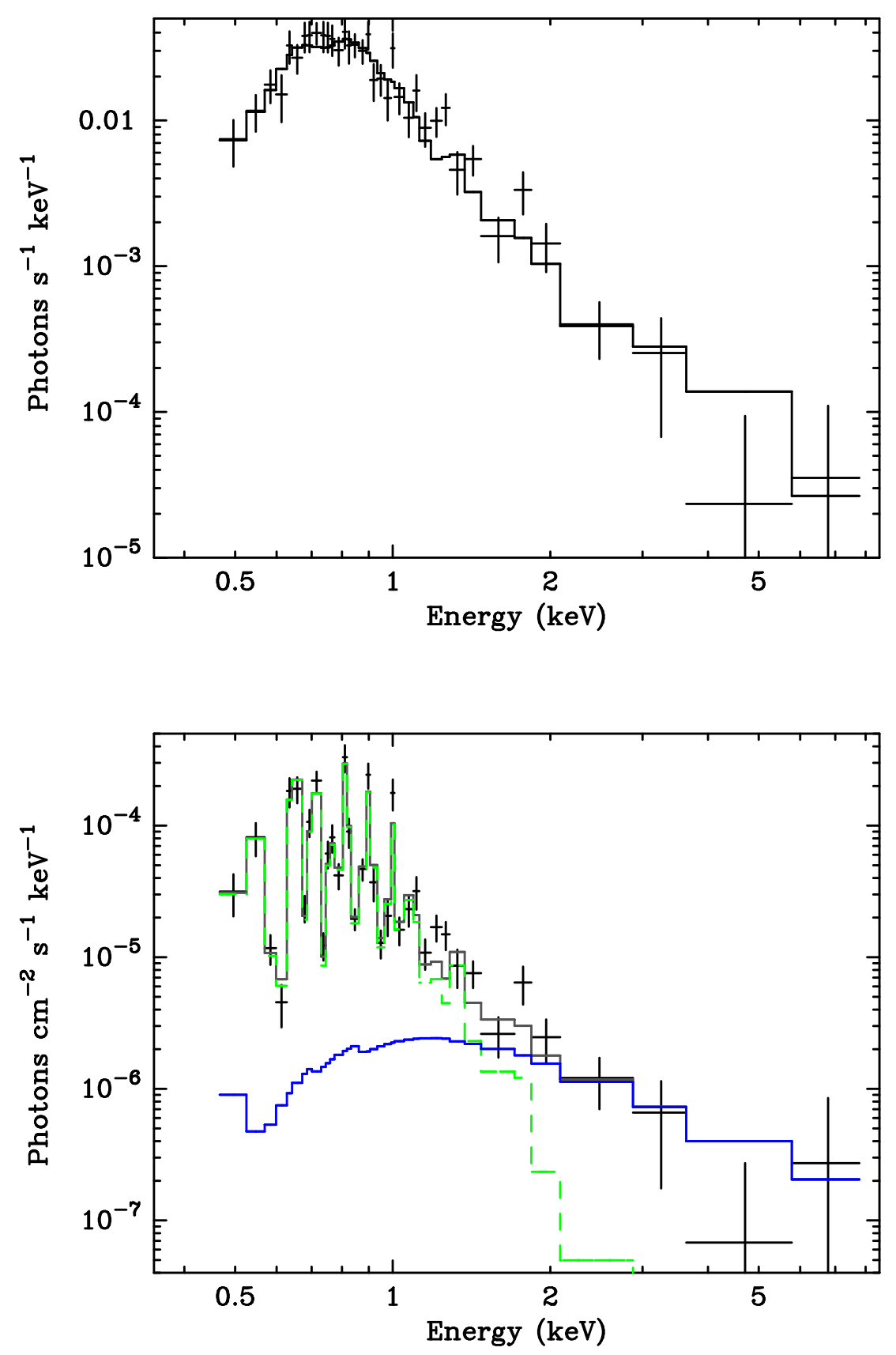

Fig. 6.- Chandra spectra of the diffuse, extra-nuclear emission of NGC 7130. (upper panel) Observed data (crosses) and total model convolved with detector response (histogram). (lower panel) Crosses show the intrinsic spectrum for the given model. The gray histogram is the total model, which requires a thermal component (green) and a hard continuum (blue). 


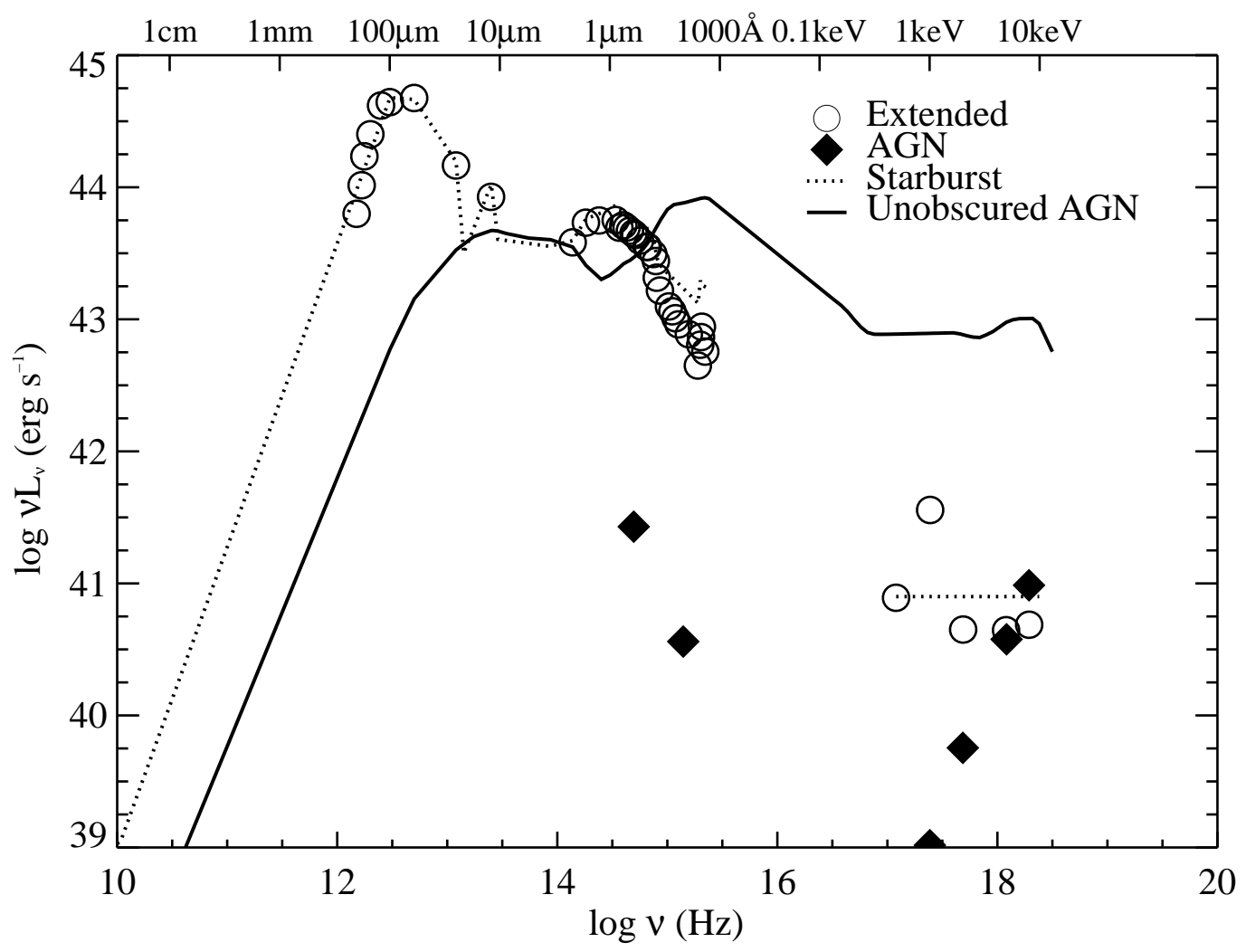

Fig. 7.- Spectral energy distributions of NGC 7130, showing large-scale emission (open circles) and the spatially and spectrally isolated AGN contributions (filled diamonds). On large scales, the galaxy appears very similar to dusty starbursts (dotted line), from Schmitt et al. (1997), with the X-ray scaling of Ranalli et al. (2003). For comparison, the average radio-quiet quasar spectrum (Elvis et al. 1994) scaled to the estimated intrinsic bolometric luminosity of NGC 7130 is also plotted (solid line). 


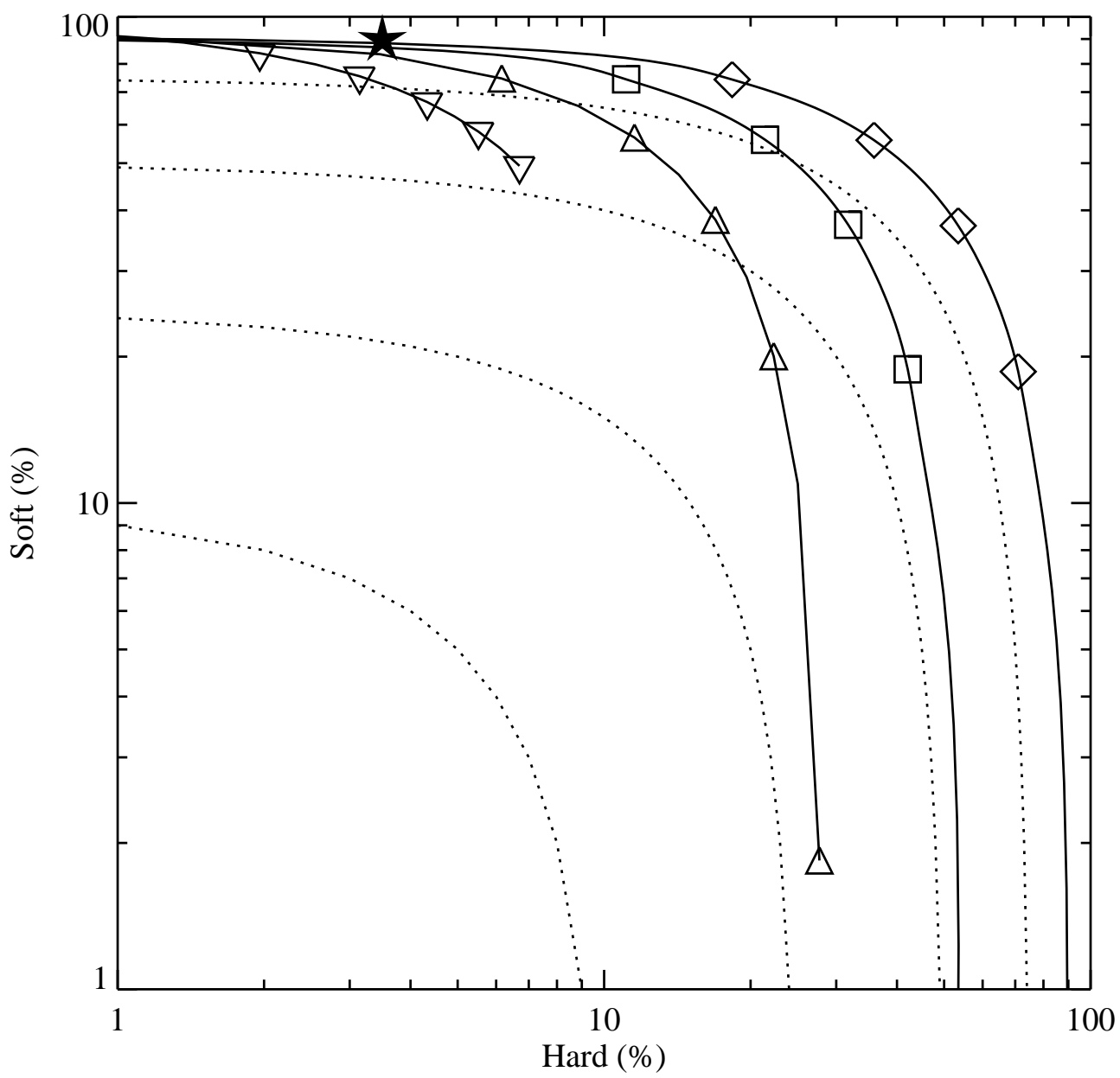

Fig. 8.- Percentage of total observed counts in the soft $(0.3-2 \mathrm{keV})$ and hard $(5-8 \mathrm{keV})$ bands as a function of AGN obscuration and starburst fraction. Along solid curves, AGN obscuration is fixed, at $N_{H}=10^{22}$ (inverted triangles), $10^{23}$ (triangles), $3 \times 10^{23}$ (squares), and $10^{24} \mathrm{~cm}^{-2}$ (diamonds). In each case, the symbols are plotted at starburst contributions of $0,20,40,60$, and $80 \%$, from lower right to upper left. A pure starburst would appear at (hard, soft $)=(0.8,93)$. Contours of constant medium-energy $(2-5 \mathrm{keV})$ contribution are plotted with dotted lines, for 90, 75, 50, and $25 \%$ from lower left to upper right. NGC 7130 is plotted as a filled star near $(4,90)$. 